Seccion: El docente y su entorno

\title{
Un reto para el profesor en la actualidad: las metodologías centradas en el aprendizaje
}

Departamento de Formación y Actualización Académica

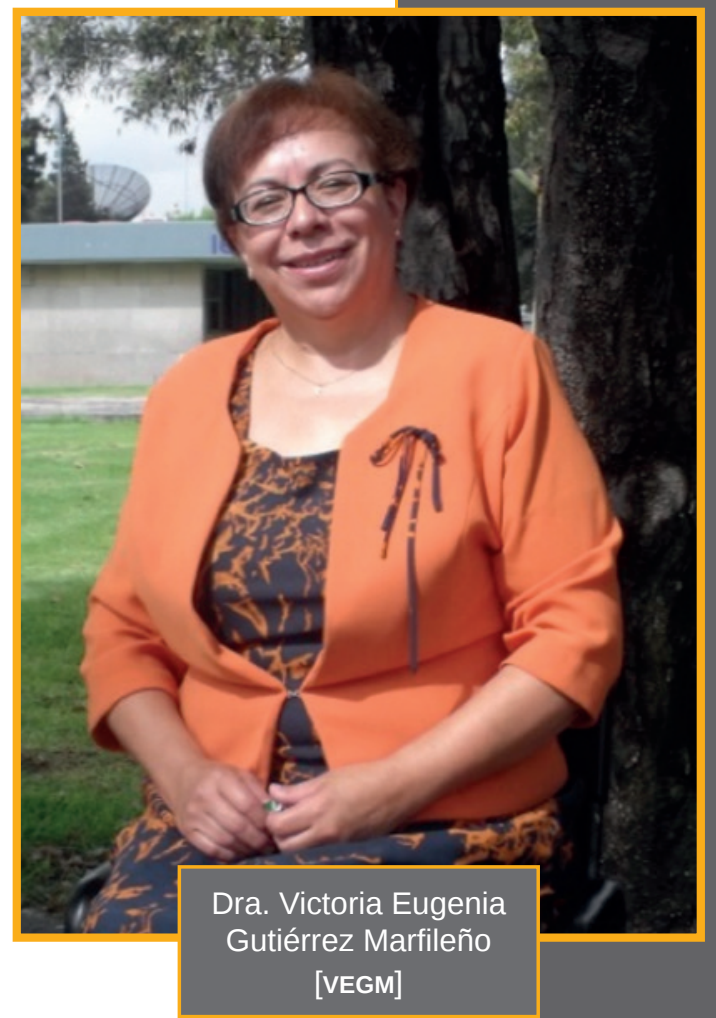

Profesora e investigadora de la Universidad Autónoma de Aguascalientes, adscrita al Centro de Ciencias Sociales y Humanidades, Departamento de Educación desde hace 25 años.

En docencia ha impartido clase tanto en licenciatura como en maestría y doctorado en las áreas de metodología de la investigación, evaluación educativa, diseño curricular y metodologías de enseñanza y aprendizaje, entre otras.

Ha sido formadora de profesores en habilidades para la planificación de la enseñanza, implementación de la misma y evaluación de los aprendizajes desde enfoques tradicionales y alternativos, en habilidades para la investigación y de manera reciente en competencias básicas para la docencia.

En investigación ha realizado distintos proyectos en las temáticas de evaluación educativa, desarrollo curricular, enseñanza y aprendizaje de la ciencia. Pertenece al cuerpo académico de "Actores y Procesos de la Educación". Actualmente, desarrolla el proyecto de investigación titulado "Aprendizaje de la Química a través de un experimento de enseñanza".

Ha asesorado más de 20 tesis de maestría, especialidad y doctorado.

Coordinó la elaboración del Modelo Educativo Institucional, el Modelo Curricular por Competencias así como el Diplomado de Formación de Competencias Básicas para la Docencia impartido en el marco de la implementación del Curriculum por Competencias del Bachillerato de la UAA del cual fue asesora en su diseño y primera fase de su implementación.

a Doctora en Educación Victoria Eugenia Gutiérrez Marfileño, en entrevista ${ }^{1}$ con el Departamento de Formación y Actualización Académica (DEFAA) nos da a conocer algunas de sus comprensiones sobre la enseñanza y nos comparte una experiencia realizada con un grupo de estudiantes implementando una metodología de enseñanza activa. Señala que se trata de métodos de enseñanza cuyos planteamientos coinciden con los realizados en el Modelo Educativo Institucional, sugiere que los profesores deben ir incorporando, de manera gradual, formas de enseñanza y evaluación del aprendizaje más acordes a las formulaciones de dicho Modelo cuyo propósito es la formación integral de los estudiantes de la UAA. 
[DEFAA]: ¿Cuál es la diferencia entre los conceptos "metodología", "método" y "técnica"?

[VEGM]: El término "método" hace referencia (como su etimología lo indica) a un camino que se sigue, un camino sistemático y ordenado para lograr un fin, en este caso, el aprendizaje. La "metodología" sería estrictamente el estudio del método; y la "técnica" tiene que ver con una serie de procedimientos de los cuales se siguen ciertos pasos de manera ordenada. En el caso de la pedagogía, de la didáctica, es más adecuado hablar de "modelos de enseñanza", de metodologías que de estos modelos se derivan; y luego de una serie de técnicas. Entonces estaríamos hablando de diferentes niveles de generalidad y de concretización de estos términos en la práctica de los profesores.

[DEFAA]: ¿Qué podemos comprender por metodología de enseñanza centrada en el aprendizaje?

[VEGM]: Básicamente, el principio de las metodologías centradas en el aprendizaje es la actividad reflexiva por parte del estudiante. Se demanda a los estudiantes no un papel pasivo en el proceso, sino una participación muy activa y reflexiva. Los profesores pensamos que cuando el estudiante asume un rol activo a nosotros nos corresponde asumir un rol pasivo, y no es así. En los métodos centrados en el aprendizaje, el papel del profesor es tan activo como el del estudiante, solamente que cambia su protagonismo en el aula y lo asume en la planificación, el diseño de la enseñanza y en la evaluación. Por un lado, el profesor tiene que crear oportunidades de aprendizaje significativas y pertinentes; además, debe dar seguimiento a los aprendizajes de los estudiantes y estar retroalimentando de manera oportuna, precisa, y señalando las maneras de trascender el error. Por eso, en los métodos activos se demanda un mayor dominio de las disciplinas que imparte el profesor, y un mayor conocimiento pedagógico.

[DEFAA]: ¿Cuántas metodologías de enseñanza conoce usted y cómo es su clasificación?
[VEGM]: Las metodologías de enseñanza se clasifican de diferentes maneras, atendiendo a los criterios que tengan de base. Hay diferentes criterios para clasificar los métodos de enseñanza: 1) por el tipo de razonamiento al que apelan o promueven en el estudiante, hay métodos deductivos vs. métodos inductivos. Otra clasificación tiene que ver con 2) las actividades que se demandan al estudiante; entonces se habla de métodos pasivos y métodos activos. Otros criterios tienen que ver con 3) la forma de organizar el curso; hay cursos o modelos que se dice están centrados en la disciplina vs. modelos centrados en la psicología del estudiante. Se habla también de métodos individuales vs. métodos socializadores. Hay incluso algunos métodos y clasificaciones en desuso como: el tipo de conocimiento al que se somete el estudiante, son los métodos heurísticos (los cuales contribuyen a la indagación y posibilidad de poner a los estudiantes en contacto con diferentes puntos de vista, que ellos sepan que la realidad es compleja y que para explicarla hay muchas formas) vs. métodos dogmáticos (aceptar el conocimiento por autoridad, y en donde existe una sola verdad: la del profesor).

Cabe señalar que los métodos no son excluyentes; es decir, el método centrado en el estudiante puede también participar de un método más hipotético-deductivo, o más inductivo. Podríamos entonces en un momento clasificar una manera de proceder para enseñar dentro de los distintos métodos.

[DEFAA]: ¿Cuál es la importancia de que el profesor conozca las metodologías de enseñanza centradas en el aprendizaje, en el contexto del Modelo Educativo Institucional (MEI) de la UAA?

[VEGM]: Uno de los rasgos que el MEI señala es que la educación que se imparta dentro de la universidad debe estar centrada en el aprendizaje del estudiante, quien será el protagonista de su propio proceso, y a quien habrá de conducirse a que logre cada vez más aprendizajes independientes. Por otro lado, cuando se señalan las características del profesor, se dice que éste tendrá la competencia o habilidad para hacer uso de métodos de instrucción óptimos a fin de lograr los aprendizajes. En ese sentido, si 
nosotros como profesores debemos estar al nivel en el que se nos demanda nuestra actuación, tendremos que buscar métodos de enseñanza o formas de enseñar que den respuesta pertinente a ese planteamiento que se señala institucionalmente; es decir, a esa aspiración educativa.

\section{[DEFAA]: ¿Cómo lograrlo?}

[VEGM]: Básicamente la formación de los profesores se da como mayormente se conoce, con los cursos de formación y actualización académica. Pero existen otras formas en las que deberíamos incursionar. En el caso del Centro de Educación Media de la UAA, cuando implementamos el $\mathrm{Cu}$ rrículo por Competencias, efectuamos una formación de los profesores in situ; es decir, en la medida en que se iba implementando el currículo nosotros asesorábamos a los profesores en la planeación de sus experiencias de aprendizaje, ofreciendo apoyo en el aula para su impartición, lo cual les resultó muy significativo.

Por otro lado, hay maneras de "autoformación" que hemos explotado poco en la institución. Por ejemplo, personas con las competencias necesarias podrían diseñar materiales de estudio independiente completo, para que los profesores los revisaran y lograran los propósitos de aprendizaje que estos contenidos tienen. Esto es, diseñar el material, las formas en las que el profesor va a interactuar con el material y algún mecanismo de evaluación.

[DEFAA]: ¿Qué elementos debería considerar el profesor para la elección de la metodología de enseñanza por aplicar?

[VEGM]: De entrada, podemos decir que no hay una metodología que lo resuelva todo ni que sea mágica; solamente es una herramienta que, junto con otras cosas, ayuda a que el profesor tenga más o menos éxito en su actividad docente. Un aspecto muy importante para considerar para decidir qué metodología es pertinente son los objetivos de aprendizaje. Hay que preguntarse a sí mismo: ¿cuál es el objetivo que pretendo alcanzar?, eso determina qué metodología se debe emplear; ¿qué tipos de contenidos de aprendizaje voy a manejar?, conocimientos, habilidades, variables afectivas, entre otros. Por otro lado, la modalidad en la que se imparta el curso. Hay que considerar que imponen ciertas condiciones al método de enseñanza que uno quiera utilizar.

El tamaño del grupo es otro aspecto a considerar; entre más centrado en el estudiante esté el método, supone un seguimiento individual mayor por parte del profesor. Entonces, habrá metodologías que sean difíciles de implementar con grupos numerosos, mas no imposibles. Hay experiencias personales, y que la literatura reporta, en las que se ha trabajado con muchos profesores (por ejemplo, diseñando portafolios) con un grupo numeroso, cuando podría pensarse que el diseño de un portafolio es con grupos pequeños; creo que también depende mucho de la habilidad del profesor.

Otro aspecto para tomar en cuenta son las demandas que la metodología impone en términos de actividad de los estudiantes. Las metodologías activas suponen ciertos principios o actividades que deben realizar los estudiantes, por ejemplo hay metodologías que invitan al trabajo colaborativo, a realizarse en escenarios naturales, o la resolución de casos; entonces, nosotros tenemos que ver cuáles son las implicaciones que se tendrían al implementarla a nuestros estudiantes. Otra consideración es la propia naturaleza de la disciplina. Hay áreas del conocimiento que se prestan más a ciertas metodologías, por ejemplo el método de estudio de caso es una aplicación muy natural en áreas como el Derecho y la Medicina.

El aprendizaje previo de los estudiantes es otra consideración que hay que hacer. Existen metodologías que suponen cierta estructura en términos de conocimiento por parte de los estudiantes.

En general, la elección de un método de enseñanza supone reflexionar sobre cuáles son los propósitos y contenidos de aprendizaje; los recursos con los que se cuenta; el número de estudiantes y la modalidad en la que la experiencia educativa va a ser impartida, en función de estos aspectos podemos elegir la estrategia más adecuada.

[DEFAA]: En la actualidad, ¿cuáles son los retos principales del docente en la implementación de las metodologías de enseñanza centradas en el aprendizaje de los estudiantes? 
[VEGM]: En primer lugar, conocerlas. Mi experiencia con profesores durante mucho tiempo es que muchas cosas no las hacen porque no las conocen. El profesor debe tener un inventario de herramientas metodológicas para enseñar, evaluar, atender a un grupo y crear ciertos climas de aprendizaje. Debe conocerlas, y una vez que se logra, hay que tratar de implementarse de manera gradual.

Hay una línea de investigación sobre profesores eficientes en donde se ha observado que entre los rasgos que caracterizan a estos docentes se encuentra el hecho de que implementan metodologías innovadoras de manera gradual y en la medida que ganan dominio implementan otras, haciéndose así de un amplio repertorio de estrategias para enseñar. Ésta es la manera más eficiente de hacerlo.

Por otro lado, el profesor debe saber que no se emplea una metodología de manera mecánica, ni acrítica (no es una receta), son grandes orientaciones, una guía para la acción pero que el profesor tiene que adecuar a su contexto de grupo, de enseñanza, hasta que vaya logrando dominio de los contenidos. Una cosa que tenemos que hacer los profesores es probar si esos métodos efectivamente son lo que la teoría dice que son. Es una función importante del profesor probar la bondad de estos métodos en términos del logro de aprendizaje.

[DEFAA]: ¿Cómo puede el docente enriquecer la metodología de enseñanza que elija aplicar para lograr el aprendizaje significativo?

[VEGM]: Las metodologías solamente son una parte de la buena enseñanza. Nos faltan otras cosas; una muy importante es la evaluación, y particularmente la evaluación formativa, la cual tiene como propósito identificar la situación de aprendizaje del estudiante. No tiene un fin de calificación, no es una evaluación sumativa sino formativa, en la que el papel del profesor es dar seguimiento a la evolución o al dominio gradual obtenido del aprendizaje, conocimiento, habilidad o actitud; retroalimentar ese aprendizaje a través de las producciones que generan los estudiantes o sus desempeños, pero retroalimentarlo con tres condiciones: precisa, oportuna y continua, de manera que el estudiante se dé cuenta cuál es el error, para lograr el dominio de ese contenido (sea una habilidad, un conocimiento o una actitud). Por otro lado, debe ofrecerle la solución para trascender dicho error; es decir, no basta decirle: "estás mal", tendría que decirle: "ahora, hay que hacer esto o aquello". La evaluación sumada a un buen modelo de instrucción logra mayores posibilidades de éxito.

Pero también, se tiene que pensar en otros aspectos como los materiales de apoyo o didácticos, los cuales son un medio de aprendizaje, una herramienta de mediación entre el estudiante y los contenidos de aprendizaje. Otro elemento que se suma a la metodología, a la evaluación y a los recursos didácticos es que el profesor sepa favorecer ambientes de aprendizaje, en donde éste tenga una mayor posibilidad de lograrse: aprendizajes respetuosos, de confianza (para preguntar, intervenir o participar), así como ambientes disciplinados para que se pueda trabajar.

Es importante tener en cuenta estos aspectos, además de la consideración a variables afectivas: qué actitudes tienen los estudiantes hacia la materia, el conocimiento, la carrera y la escuela; cuáles son sus intereses, entre otras. El aprendizaje es una variable compleja que se ve impactada por muchas otras. En la medida en que el profesor conozca cuáles son esas y cómo las puede ir controlando, garantiza que el aprendizaje sea duradero, pertinente y útil. Esto es, que resulte "significativo" para el estudiante.

[DEFAA]: ¿Puede describir alguna experiencia que considere haya sido exitosa al aplicar alguna metodología de enseñanza?

[VEGM]: El semestre pasado se trató con esta idea de que los profesores probáramos lo que propone un método activo de implementar una experiencia educativa. Se empleó un método de proyectos con los estudiantes de tercer semestre de la Licenciatura en Docencia del Idioma Inglés (LDII) de la UAA, en la materia de Diseño Curricular. El proyecto consistió en que ellos diseñaran un plan de estudios para apoyar el Programa de Movilidad de Estudiantes que existe en la misma institución. Se les planteó inicialmente como si fuese un proyecto real; se les dijo que la institución les solicitaba a los es- 
tudiantes de la LDII que diseñaran un plan de estudios para los alumnos que se van a un país de habla inglesa, y que requieren desarrollar esta competencia comunicativa. Ellos realizaron todo el proceso de diseño curricular, desde el apartado de fundamentación, donde muestran la pertinencia, la relevancia y la factibilidad de un programa; investigaron con qué infraestructura contaba el Departamento de Idiomas de la UAA, cuántos profesores, qué tan calificados y con qué recursos disponía el Centro de Aprendizaje Autodirigido de Idiomas (CAADI); todo eso para ver si el programa era viable. Después, investigaron programas que se ofrecen en apoyo a los estudiantes de movilidad para determinar los contenidos de aprendizaje. Luego se revisaron referentes como el Marco Común de Referencia para las Lenguas Extranjeras; de ahí se definieron los niveles de dominio en las distintas habilidades comunicativas. Posteriormente, diseñaron el plan de estudios (en su organización con "tantos" cursos, con "tantos" créditos y "tantas" horas), así como la evaluación del programa; es decir, ¿cómo evaluar el nivel de impacto del programa? Los estudiantes fungieron como Comité de Diseño Curricular, hicieron cuestionarios, consultaron tanto fuentes impresas como vivas, entre otras.

Fue un trabajo muy bien realizado, superó la expectativa que personalmente tenía sobre el punto. A propósito de todo esto, en ese "hacer significativo" para ellos, ya les era muy normal hablar de modelos de diseño curricular: modelo por competencias, por objetivos; las fases del diseño curricular, en qué consiste cada fase y cuál es su propósito, por qué el conocimiento se vuelve una herramienta para "hacer". El conocimiento no es el fin último del curso, sino que es un vehículo para trabajar lo que se quería hacer.

Se probó en esa experiencia que los métodos activos facilitan el alcance de los propósitos de aprendizaje. Eso no quiere decir que sea muy sencillo; es difícil porque se pierde un poco el control del grupo y se invierte mucho tiempo, los estudiantes marchan a diferentes ritmos y al profesor controlar eso le demanda mucha tensión y seguimiento. Las necesidades de información van siendo distintas, y uno como docente tiene que estar tratando de satisfacerlas, porque dentro de esa experien- cia unos estudiantes diseñaron por objetivos y otros por competencias. Por eso señalo que a los profesores los métodos activos nos demandan un mayor dominio de la materia. Fue una experiencia agradable, tiene uno que sopesar muchas cosas a favor y en contra, pero yo creo que los estudiantes quedaron satisfechos y se lograron los propósitos establecidos.

[DEFAA]: ¿Cuál fue su aprendizaje más significativo como docente a partir de la anterior experiencia?

[VEGM]: Probé algunos postulados de estos métodos. Por ejemplo, que suponen un trabajo muy activo por parte de los estudiantes: tienen que investigar, recuperar información pertinente, sintetizarla y presentarla adecuadamente. Los estudiantes se motivan más cuando hacen, que cuando solamente leen o reciben. Cuando los estudiantes le ven una utilidad a lo que están haciendo, les resulta muy significativo. Otro aprendizaje fue que, efectivamente, el profesor tiene mucho trabajo de diseño (yo tuve que diseñar el proyecto, materiales que iban a ocupar, y que ellos tuvieran la información necesaria). Tuve que dar seguimiento a cada equipo con un nivel diferenciado de avance a fin de estarles retroalimentando de manera permanente. Eso es muy desgastante porque son cosas diferentes. Y luego, tener la preocupación de que trabajen todos. Se trata entonces de estar buscando formas, de observar que todos participen y en qué nivel. Como docentes, debemos tratar de favorecer la participación de todos.

Probé algunos postulados de estos modelos activos: son ciertos, se logran algunos aprendizajes, demandan mucho trabajo para el profesor, suponen aprendizajes previos de los estudiantes, se favorece la metacognición y el aprendizaje cooperativo, se les ubica en una situación de contexto real de lo que ellos pueden hacer profesionalmente, lo que resulta motivador. Y una lección importante sería: sí lo volvería a hacer, pero ahora voy a tratar de seguir probando, adecuando, implementando otros métodos pero gradualmente.

[DEFAA]: ¿Alguna reflexión o algo que quisiera compartir a los docentes que nos leen 
respecto a la implementación de las metodologías de enseñanza centradas en el aprendizaje del estudiante?

[VEGM]: Los profesores debemos implementar estas formas de aprender en las que el estudiante se involucre de una manera reflexiva, consciente; con actividades muy pertinentes a su desempeño profesional futuro, y eso lo proporcionan este tipo de metodologías activas. Su diseño subyace estas intenciones de aprendizaje colaborativo, aprendizaje independiente (independencia del profesor), aprender a aprender, reflexión de lo que se hace y de lo que se aprende.

Es importante que vayamos implementando estas metodologías que demandan tiempo, conocimiento de cómo se realizan, cuál es su secuencia y qué actividades suponen para los estudiantes; asimismo, es esencial que nosotros [junto con los elementos que señalé anteriormente (la evaluación formativa, los recursos didácticos...) todos esos elementos para una enseñanza eficiente] atendamos todo esto en cada curso y lo tomemos cada vez como un experimento, pues tenemos un gran laboratorio en el aula.

A veces, parece que los profesores partimos de cero, damos un curso y pareciera que fuera la primera vez que lo vamos a dar. Tenemos varios años de ser docentes y no tenemos un inventario de instrumentos de evaluación, de recursos didácticos, de experiencias de aprendizaje, vamos desechando toda la experiencia buena y mala que vamos acumulando, y no debería ser así. Debemos sistematizar nuestra enseñanza. Cada curso, cada materia que impartimos debe ser una lección para la mejora del siguiente. Si vamos haciendo eso, llegaremos a ser efectivamente expertos.

La experiencia tiene que ver con hacer las cosas cada vez mejor, y solamente las hacemos cada vez mejor si nos atrevemos a innovar, a probar cosas diferentes, y si sistematizamos nuestra experiencia. Invitaría a los profesores a implementar de manera gradual estos métodos; tratar de ir considerando estas variables y ver cada experiencia de un curso como un experimento atractivo, obviamente todo en función del aprendizaje de los estudiantes. 